\title{
Magnetic helicity transported by flux emergence and shuffling motions
}

\author{
Y. Zhang ${ }^{1}{ }^{2}$, R. Kitai ${ }^{2}$ and K. Takizawa ${ }^{2}$ \\ ${ }^{1}$ Key Laboratory of Solar Activity, \\ National Astronomical Observatories of Chinese Academy of Sciences \\ email: zhangyin@bao.ac.cn \\ ${ }^{2}$ Kwasan and Hida Observatories, Kyoto University, Yamashina-ku, Kyoto 607-8471 \\ email: takizawa@kwasan.kyoto-u.ac.jp \\ kitai@kwasan.kyoto-u.ac.jp
}

\begin{abstract}
Magnetic helicity can be transported from sub-photosphere into corona by the emergence of helical magnetic field lines and the shuffling motions of foot-points of pre-existing coronal field lines. Active region NOAA 10930 was observed by SP and NFI of SOT on board Hinode when it pass through the solar meridian. Based on these observations, we calculate magnetic helicity flow of both terms, by regarding Doppler velocity as normal velocity. The results are compared with which calculated by method proposed by Zhang et. al. (2012). Our results show that helicity injection maps calculated by both methods have similar distribution and the integration values have the same magnitude.
\end{abstract}

Keywords. sun: activity, sun: photosphere, sun: surface magnetism

\section{Introduction}

Magnetic helicity can be transported from sub-photosphere into corona by the emergence of helical magnetic field lines and the shuffling motions of foot-points of pre-existing coronal field lines. (Berger \& Field 1984). The emergence of twisted fluxes and shuffling motions are also the popular triggers for solar eruptions and the common mechanism for magnetic helicity accumulation both in theoretical and observational works (Leka et al. 1996; Fan \& Gibson 2004). According to Berger (1999), the transport rate of relative helicity, $\dot{H}$, due to both processes are given by $\dot{H}_{n}=\oint 2\left(\boldsymbol{B}_{t} \cdot \boldsymbol{A}_{p}\right) v_{n} d S$ and $\dot{H}_{t}=-\oint 2\left(\boldsymbol{v}_{t} \cdot \boldsymbol{A}_{p}\right) B_{n} d S$. How to get $v_{n}$ is the key point in calculating $\dot{H}_{n}$. From the observational view, if the active region locates around the solar disk center, we can use Doppler velocity as $v_{n}$ (method 1). On the other hand, Zhang et al.(2012) proposed a method to do this calculation based on the observations of vector magnetograms and correspondence horizontal motions (method 2). Here, we compared the results obtained by both methods of an isolated active region NOAA 10930 when it passed though the solar meridian. The observational data and results will be presented in Section 2 .

\section{Observational data and results}

Data used here are obtained by the Spectro-Polarimeter (SP) (Kosugi et al. 2007; Suematsu et al. 2007; Ichimoto et al. 2007) and the Narrowband Filter Imager (NFI) of Solar Optical Telescope (SOT) (Tsuneta et al. 2008) on board Hinode. Vector magnetograms at 03:10 UT on December 112006 (Fig 1a) are derived from the inversion of full Stokes profiles, based on the assumption of Milne-Eddington (ME) atmosphere model. The horizontal motions (Fig 1b) $\boldsymbol{\mu}_{t}$ were calculated by 2 minutes longitudinal magnetograms by 


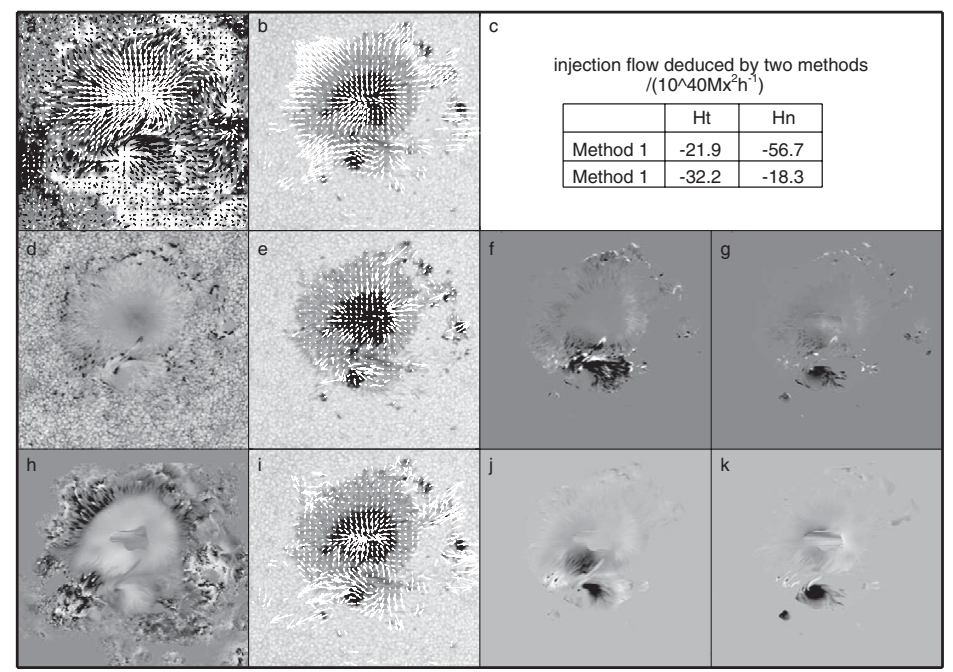

Figure 1. (a) Vector magnetogram; (b) $\boldsymbol{\mu}_{t}$; (c) table of integration values of helicity flow calculated by both methods; (d) Doppler velocity; (e)-(g) $\boldsymbol{v}_{t}$, map of helicity flow due to the vertical and shuffling motion deduced by method $1 ;(\mathrm{h})-(\mathrm{k}) \boldsymbol{v}_{\perp n}, \boldsymbol{v}_{\perp t}$, map of helicity flow due to the vertical and shuffling motion deduced by method 2 .

LCT method. Doppler map is shown in Fig 1d. Shuffling motions, deduced from horizontal motion and Doppler velocity by $\boldsymbol{v}_{t}=\boldsymbol{\mu}_{t}+\frac{v_{n}}{B_{n}} \boldsymbol{B}_{t}$ (Démoulin \& Berger, 2003), are shown in Fig 1e. Corresponding maps of $\dot{H}_{n}$ and $\dot{H}_{t}$ are shown in Fig 1 f and $1 \mathrm{~g}$. As shown in Fig 1c, the integration values of $\dot{H}_{n}$ and $\dot{H}_{t}$ are $-21.9 \times 10^{40} M x^{2} \cdot h^{-1}$ and $-56.7 \times 10^{40} M x^{2} \cdot h^{-1}$, respectively. Normal and shuffling motions deduced by method 2 are shown in Fig $1 \mathrm{~h}$ and 1i. Corresponding helicity flow maps are shown in Fig $1 \mathrm{j}$ and $1 \mathrm{k}$. The integration values of $\dot{H}_{n}$ and $\dot{H}_{t}$ are $-32.2 \times 10^{40} M x^{2} \cdot h^{-1}$ and $-18.3 \times 10^{40} M x^{2} \cdot h^{-1}$. Helicity injection map of $\dot{H}_{n}$ and $\dot{H}_{t}$, which calculated by both methods, show that the helicity injection is predominantly negative, which is consistent with the left handed twisted magnetic field (Fig 1a) and counterclockwise rotation of the sunspot (Fig 1b). Meanwhile, positive helicity injection can be found along the neutral line in $\dot{H}_{n}$ map and at the west limb of the leading sunspot in $\dot{H}_{t}$ map. The integration values are shown in Fig 1c, which show helicity injection calculated by both methods have the same magnitude. Our results demonstrate that both methods can be used to estimate the helicity injection when the active region locates around the solar disk center.

\section{References}

Berger, M. A. \& Field, G. B. 1984, J.Fluid Mech, 147,133

Berger, M. A. 1999, in Magnetic Helicity in Space and Laboratory Plasmas, ed. M. R. Brown, R. C. Canfield, \& A. A. Pevtsov (Geophys. Monogr. 111; Demoulin, P., Mandrini, C. H., van DrielGesztelyi, L., Thompson, B. J., Washington: AGU), 1

Démoulin, P. \& Berger, M. A. 2003, Solar Phys., 215, 203

Fan, Y. H. \& Gibson, S. E. 2004, ApJ, 609, 1123

Ichimoto, K., et al. 2008, Solar Phys., 249, 233

Kosugi, T., et al. 2007, Solar Phys., 243, 3

Leka, K. D., Canfield, R. C., \& McClymont, A. N. 1996, ApJ, 462, 547

Suematsu, Y., et al. 2008, Solar Phys., 249, 197

Tsuneta, S., et al. 2008, Solar Phys., 249, 167

Zhang, Y., Kitai, R., \& Takizawa, K. 2012, ApJ, 751, 85 